lesser amounts of calm, happiness, frustration, worry, or sadness than other activities by either husbands or wives. Other types of caregiving activities (by type of activity and type of recipient) will be examined.

\section{MARITAL CHARACTERISTICS AND COUPLES' SHARED TIME DURING ADULTHOOD}

S. Flood, K. Genadek, Minnesota Population Center, Minneapolis, Minnesota

This paper leverages unique data from the 2009 and 2013 Supplements on Disability and Use of Time to the Panel Study of Income Dynamics (PSID) to investigate the relationship between marital characteristics and couples' shared time during later adulthood. Previous research indicates that spousal interaction is a key dimension of marital quality. We extend previous research that has been primarily focused on time spent with a spouse for working-age adults and parents by considering couples' shared time during the stage of life after the career- and family-building years, specifically ages 50 and 79. By using the PSID, we make two key contributions to the literature. First, we analyze the quality of time with a spouse by differentiating between active and passive shared time. Second, we examine how marital quality and marital duration are associated with time with a spouse, which has been a major limitation of previous research in this area. Results show that the nature of time with a spouse - active versus passive - varies by levels of marital support and marital strain and that in the cross section, marital duration shows a u-shaped relationship with active shared time.

\section{DISABILITY AND PATTERNS OF LEISURE PARTICIPATION ACROSS THE LIFE COURSE}

C. Shandra, State University of New York at Stony Brook, Stony Brook, New York

I use nationally representative data from the Well-Being Module of the American Time Use Survey $(\mathrm{N}=38,547)$ to examine: (1) how the presence of a sensory, cognitive, physical, or multiple disability associates with the likelihood and intensity of participation in six types of leisure activities (passive, exercise, organizational, hobbies, arts, and social), and (2) if these patterns vary by age. Overall, people with all types of disability are more likely to engage in passive forms - and less likely to engage in active forms-of leisure activity; however, many of these differences are mediated by health status. These associations persist, net of sociodemographic and health controls, for people with physical or multiple disabilities. Furthermore, greater differences in leisure time by disability status are observed with increasing age. Older adults with disabilities are more susceptible to disengaged leisure time, compared both to those without disability and to younger adults with disability.

\section{SESSION 130 (SYMPOSIUM)}

\section{FACILITATING PURPOSE IN LATER LIFE}

Chair: J. Nakamura, Claremont Graduate University, Claremont, California

Discussant: L.L. Carstensen, Stanford University

Across the life course, dedication to a social purpose is associated with both individual and collective well-being. Increasingly, later life is being recognized as a period when adults may form a purpose goal meaningful to themselves and useful to others and dedicate their energies to pursuing it, for example, by leveraging accrued experience to create new ways of addressing social problems. Three papers will share empirical reports addressing how older adults in the U.S. form and pursue social purpose and the psychosocial and institutional means by which this can be facilitated, summarizing lessons learned from three mixed-methods research projects. A leader in the psychology of aging will provide a discussion. Anne Colby will present results of a study of purpose beyond-the-self and its correlates in adults over 50 , based on a survey of a nationally representative sample $(n=1,200)$ and over 100 interviews on the topic. Jeanne Nakamura will present a paper based on 50 in-depth interviews with individuals over 60 who have been honored for successful social innovation, charting the diverse pathways those individuals took to realize their purpose goals. Jim Emerman will discuss results of a survey of organizations helping older individuals find ways to live out their purpose goals, mapping programmatic resources available to this population and analyzing opportunities and gaps in services. Then, Laura Carstensen will provide an integrative discussion of the papers, informed by her extensive body of work. The session will conclude with discussion by the panel and audience of issues that have been raised.

\section{NATIONAL STUDY OF PURPOSE BEYOND THE SELF IN OLDER ADULTS}

A. Colby ${ }^{1}$, M. Bundick ${ }^{2}$, K. Remington ${ }^{1}$, 1. Stanford

University, Palo Alto, California, 2. Duquesne University,

Pittsburgh, Pennsylvania

This paper reports on the findings of a study of purpose beyond-the-self (bts) in U.S. adults aged 50-90. Purpose beyond-the-self is defined as active engagement toward goals that are meaningful to the self and contribute to the world beyond the self. The study includes a nationally representative survey of 1,200 respondents, with in-depth interviews of 107 . Analyses to date indicate that about $30 \%$ of older adults exhibit purpose bts; that purpose is approximately equally prevalent across age, gender, education, income, and health status; and that people of color are more likely to be purposeful than are white respondents. Further analyses will report the significant positive relationships between purpose and other indicators of positive adaptation, including gratitude, prosocialness, generativity, and personal growth initiative. The nature, significance, and dynamics of purpose in later life will be explicated through interview-based case examples, describing highly purposeful individuals from a wide range of life situations.

\section{PATHWAYS TO SOCIAL PURPOSE AND INNOVATION AFTER SIXTY}

J. Nakamura, L. Graham, T. Chan, K. Procter, Psychology, Claremont Graduate University, Claremont, California

Much attention has been devoted to traditional forms of social contribution in later life such as volunteering. In contrast, little is known about social innovation - the creation of new ways to address persistent social problems. In particular, the pathways leading to social innovation in later life are largely uncharted. In an ongoing mixed-methods research project, we are examining the nature, antecedents, 